\title{
Electron Transfer Dissociation of Peptide Anions
}

\author{
Joshua J. Coon, Jeffrey Shabanowitz, and Donald F. Hunt ${ }^{\dagger}$ \\ Department of Chemistry, University of Virginia, Charlottesville, Virginia, USA \\ John E. P. Syka* \\ Engineering Physics Program, University of Virginia, Charlottesville, Virginia, USA
}

Ion/ion reactions of multiply deprotonated peptide anions with xenon radical cations result in electron abstraction to generate charge-reduced peptide anions containing a free-radical site. Peptide backbone cleavage then occurs by hydrogen radical abstraction from a backbone amide $\mathrm{N}$ to facilitate cleavage of the adjacent $\mathrm{C}-\mathrm{C}$ bond, thereby producing a- and $\mathrm{x}$-type product ions. Introduction of free-radical sites to multiply charged peptides allows access to new fragmentation pathways that are otherwise too costly (e.g., lowers activation energies). Further, ion/ion chemistry, namely electron transfer reactions, presents a rapid and efficient means of generating odd-electron multiply charged peptides; these reactions can be used for studying gas-phase chemistries and for peptide sequence analysis. (J Am Soc Mass Spectrom 2005, 16, 880-882) @ 2005 American Society for Mass Spectrometry

$\mathrm{R}$ ecently our laboratory described electron transfer reactions of multiply protonated peptide cations with singly charged polynuclear aromatic anions to induce extensive peptide backbone fragmentation, electron transfer dissociation (ETD) [1, 2]. In a sense, ETD is the ion/ion analog of electron capture dissociation [3] - the reagent anion is simply a vehicle for electron delivery. In much earlier work, McLuckey and coworkers reported gas-phase electron transfer reactions of multiply deprotonated nucleic acids with rare gas cations [4]. Electron removal from the olignonucleotide anion generated both charge-reduced precursor and low-level fragment ions that were not observed following collision-activated dissociation (CAD) [4-6]. In a later ion/electron experiment, Zubarev et al. bombarded multiply deprotonated peptide anions with electrons $(>10 \mathrm{eV})$ to induce electron detachment from the peptide and subsequent backbone fragmentation (electron detachment dissociation, EDD) [7]. Although McLuckey and colleagues did not apply the ion/ion analog of EDD to peptide anions, their work with nucleic acids suggests electron transfer induced dissociation of the peptide backbone would likely follow.

Published online April 14, 2005

Dedicated to John R. Yates, III, Recipient of the 2004 Biemann Medal.

Address reprint requests to Dr. J. J. Coon, Department of Chemistry, University of Virginia, McCormick Road, Charlottesville, VA 22908, USA. E-mail: jcoon@virginia.edu

* Also at Thermo Electron, 355 River Oaks Parkway, San Jose, CA 95134. + Also at the Department of Pathology, Health Sciences Center, University of Virginia, Charlottesville, VA 22908.

\section{Results and Discussion}

Using a modified quadrupole linear ion trap (QLT) mass spectrometer [1,2], we have examined the reactions of multiply deprotonated peptides with cations. The $200 \mathrm{~ms}$ reaction of the triply deprotonated phosphopeptide, LPISASHpSpSKTR, with radical cations of xenon generates extensive fragmentation with concomitant charge reduction (Figure 1). Of the dissociation products, a- and x-type fragment ions are most prevalent. Losses of carbon dioxide, phosphoric acid, and multiple combinations thereof are observed from both the charge-reduced precursor and the a- and x-type fragments. CAD, on the other hand, mainly produces a doubly deprotonated product corresponding to loss of a phosphate modification $\left(\mathrm{HPO}_{3}^{-},>95 \%\right)$.

Electron abstraction by $\mathrm{Xe}^{+}$generates a radical containing charge-reduced peptide capable of dissociation driven by free-radical chemistry. Figure 2 presents a possible mechanism describing the a- and x-type fragmentation observed in Figure 1. Here we illustrate this mechanism by following the reaction of the triply deprotonated peptide, $X_{n} E A$, with the Xe cation. The ion/ion reaction results in formation of a charge-reduced peptide that contains a radical site on the carboxyl group of the second residue. Abstraction of a hydrogen radical from a backbone amide $\mathrm{N}$ facilitates cleavage of the adjacent $\mathrm{C}-\mathrm{C}$ bond to produce a- and $\mathrm{x}$-type product ions.

In addition to the a- and x-type products, we also note a series of ions having mass-to-charge ratios 44 units lower than those contained in the x-type fragment series (Figure 1). We suggest the $x-44$ ions arise from 

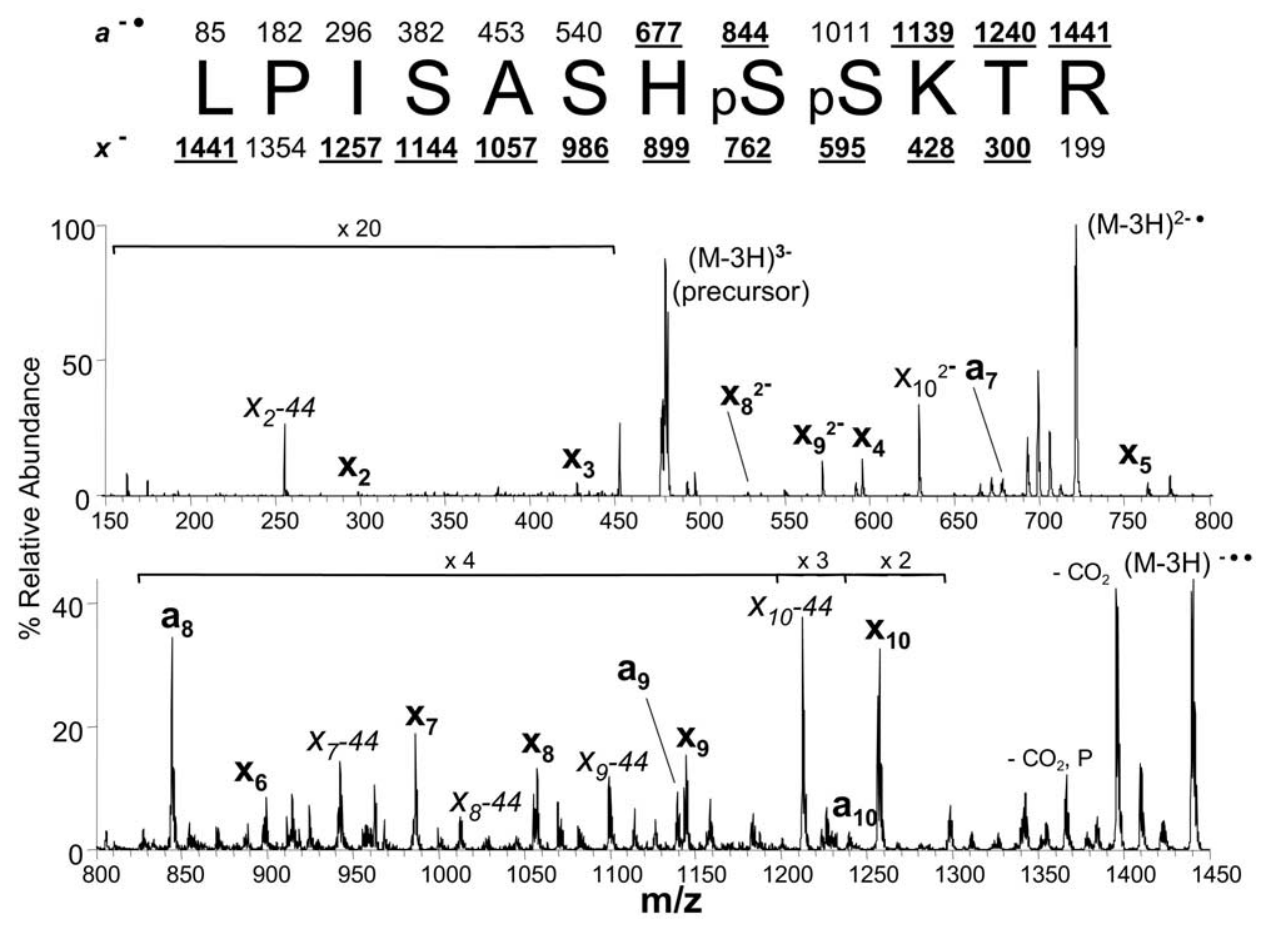

Figure 1. Tandem mass spectra resulting from a $200 \mathrm{~ms}$ reaction of the triply deprotonated phosphopeptide, LPISASHpSpSKTR, with radical cations of Xe (average 10 single-scan mass spectra).

multiply deprotonated peptides that have lost at least two electrons to $\mathrm{Xe}^{+}$. One electron removal likely occurs at the c-terminus, wherein free-radical-driven chemistry triggers neutral loss of $\mathrm{CO}_{2}$, while another induces peptide backbone cleavage to generate an $x$ type fragment, which has previously lost its carboxy terminus $\left(\mathrm{x}_{\mathrm{n}}^{-}-44\right.$, note these processes could occur in either order). If, for example, the $x_{2}^{2-}$ product ion of the triply deprotonated $X_{n} E A$ peptide were to lose an electron through a subsequent reaction with $\mathrm{Xe}^{+}$, neu-<smiles>[R]NC(CCC(=O)[O-])C(=O)NC(C)C(=O)[O-]</smiles>

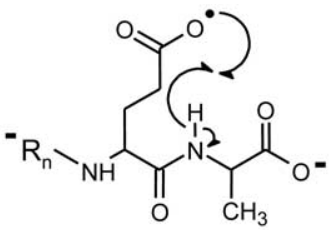<smiles>[R]N[C](CCCC)CCC(=O)O</smiles><smiles>[R]NC1CCN(C(C)C(=O)[O-])C(=O)C1CCC(=O)O</smiles>

Figure 2. Fragmentation scheme for production of a- and x-type ions following electron abstraction by Xe cations from a multiply deprotonated peptide, $\mathrm{X}_{\mathrm{n}} \mathrm{EA}$ (note, uppercase $\mathrm{X}$ is used to represent any amino acid). tral loss of $\mathrm{CO}_{2}$ could be triggered to generate an $\mathrm{x}_{2}^{-\cdot}-$ $\mathrm{CO}_{2}$ product (Figure 3). Inspection of the tandem mass spectrum generated following reaction of the doubly deprotonated phosphopeptide with Xe cations (here the observed products could only result from a single electron transfer event) showed markedly reduced production of the $x_{n}-C_{2}$-type product ions (two $x_{n}-$ $\mathrm{CO}_{2}$ products were observed at $\sim 5 \%$ abundance relative to their respective $x$-type fragment, data not shown).

Our results are, to a certain extent, comparable with those achieved via EDD by Zubarev and coworkers for the doubly deprotonated caerulein peptide [7]. For example, they also noted neutral losses of $\mathrm{CO}_{2}$ from both the precursor and numerous fragments. Their experiment, however, produced a-, c-, and z-type fragment ions, while our ion/ion reaction predominately generated formation of a- and x-type fragments, with few or no c- and z-type fragments (from a limited set of other peptides, data not shown). It is conceivable that<smiles>[Y4]C(C)C(=O)NC(=O)C(CCC(=O)[O-])N=C=O</smiles>

Figure 3. Fragmentation scheme for production of $\mathrm{x}_{\mathrm{n}}^{-\cdot}-\mathrm{CO}_{2}$ resulting from multiple electron abstraction events. 
energetic electrons may be less selective in electron abstraction than the Xe cations used here. Any diversity in the initial location of the removed electron could allow EDD to access different reaction pathways than those achieved with negative electron transfer dissociation (NETD). Further characterization of both electron detachment approaches will be required to reveal these and other possible differences between the methods.

Use of Xe cations for electron abstraction precludes the proton transfer side reaction; note that proton transfer to peptide anions is highly exothermic [6]. To study proton transfer reactions of peptide anions, we introduced another cation-protonated fluoranthene. The proton transfer reaction mainly reduced the charge of the peptide (eq 1), with only a small amount of fragmentation, $\mathrm{CO}_{2}$ loss $(\sim 5 \%$ relative abundance, data not shown).

$$
\mathrm{C}_{16} \mathrm{H}_{11}^{+}+[\mathrm{M}-3 \mathrm{H}]^{3-} \rightarrow \mathrm{C}_{16} \mathrm{H}_{10}+[\mathrm{M}-2 \mathrm{H}]^{2-}
$$

Examination of the isotopic distribution of the chargereduced peptide anion revealed electron transfer still occurred to a minor extent; the transfer presumably occurred from either fluoranthene or a low-level background cation. In either case, since the neutral loss product ion was 45 units less than the charge-reduced proton transfer product, we conclude the $\mathrm{CO}_{2}$ loss was triggered by the electron transfer side reaction.

$\mathrm{Wu}$ and McLuckey describe both electron and proton transfer reactions for oligonucleotide anions with fragmentation most prevalent following the former pathway (a result in agreement with our observations for peptide anions). Calculations prompted those authors to conclude that reaction exothermicity-not radical site introduction-was the major driving force for nucleotide anion fragmentation following ion/ion reaction [6]. In another experiment Zubarev et al. reacted peptide anions with $\mathrm{H}^{+}$ cations and observed neither $\mathrm{CO}_{2}$ loss nor cleavage of the backbone, stimulating them to suggest that polypeptide anions are only facile when a radical site is present [7]. At least for peptides, we find our results consistent with Zubarev's assertion that electron removal from peptide anions induces fragmentation driven by free-radical chemistry. In short, introduction of free-radical sites to multiply charged peptides allows access to new fragmentation pathways that are otherwise too costly (e.g., lowers activation energies). Ion/ion chemistry, namely electron transfer reactions, presents a rapid and efficient means of generating odd-electron multiply charged peptides. These reactions can be used for studying gas-phase chemistries and for peptide sequence analysis.

\section{Conclusions}

Protein phosphorylation is arguably the most important and common post-translational modification, with a key role in signal transduction and amplification [8]. Today, phosphopeptide sequence analysis by tandem mass spectrometry is almost exclusively carried out by:
(1) generation of peptide cations and (2) fragmentation using CAD. Phosphopeptide analysis has remained challenging because, compared with their unphosphorylated counterparts, they are present at lower abundances, do not ionize as efficiently (in the positive mode), and routinely dissociate uninformatively via CAD. Phosphopeptides do, however, ionize preferentially under negative electrospray (NESI) conditions, owing to the acidic phosphate moiety. In fact, Carr and coworkers have developed an entire methodology based on NESI and single ion monitoring $\left(\mathrm{PO}_{2}^{-}\right.$and $\mathrm{PO}_{3}^{-}$) for phosphopeptide detection $[9,10]$. Following detection, the corresponding positive ions (the intact peptide ion) are fragmented, using $\mathrm{CAD}$, for sequence analysis and phosphorylation site location. With NETD, we envision possibilities for direct phosphopeptide detection and characterization in the most sensitive format: negative ionization. Further, because nonacidic peptides will be suppressed in the negative ion mode, NETD may obviate the need for phosphopeptide enrichment strategies and/or may allow characterization of previously inaccessible acidic peptides/proteins.

\section{Acknowledgments}

The authors acknowledge the National Institutes of Health (DFH, GM37537, and AI33993; JJC, RR018688), the National Science Foundation (DFH, MCB-0209793), and Thermo Electron for their support. They also thank Jim Stephenson and Roman Zubarev for helpful discussions.

\section{References}

1. Syka, J. E. P.; Coon, J. J.; Schroeder, M. J.; Shabanowitz, J.; Hunt, D. F. Peptide and protein sequence analysis by electron transfer dissociation mass spectrometry; Proceedings of the National Academy of Sciences of the United States of America; 2004; pp 9528-9533.

2. Coon, J. J.; Syka, J. E. P.; Schwartz, J. C.; Shabanowitz, J.; Hunt, D. F. Anion dependence in the partitioning between proton and electron transfer in ion/ion reactions. Int. J. Mass Spectrom. 2004, 236, 33-42.

3. Zubarev, R. A.; Kelleher, N. L.; McLafferty, F. W. Electron capture dissociation of multiply charged protein cations. A nonergodic process. J. Am. Chem. Soc. 1998, 120, 3265-3266.

4. Herron, W. J.; Goeringer, D. E.; Mcluckey, S. A. Gas-phase electron-transfer reactions from multiply charged anions to rare-gas cations. J. Am. Chem. Soc. 1995, 117, 11555-11562.

5. Stephenson, J. L.; McLuckey, S. A. Charge reduction of oligonucleotide anions via gas-phase electron transfer to xenon cations. Rapid Commun. Mass Spectrom. 1997, 11, 875-880.

6. Wu, J.; McLuckey, S. A. Ion/ion reactions of multiply charged nucleic acid anions: Electron transfer, proton transfer, and ion attachment. Int. J. Mass Spectrom. 2003, 228, 577-597.

7. Budnik, B. A.; Haselmann, K. F.; Zubarev, R. A. Electron detachment dissociation of peptide di-anions: An electron-hole recombination phenomenon. Chem. Phys. Lett. 2001, 342, 299-302.

8. Hunter, T. Signaling-2000 and beyond. Cell 2000, 100, 113-127.

9. Huddleston, M. J.; Annan, R. S.; Bean, M. F.; Carr, S. A. Selective detection of phosphopeptides in complex-mixtures by electrospray liquid-chromatography mass-spectrometry. J. Am. Soc. Mass Spectrom. 1993, 4, 710-717.

10. Annan, R. S.; Huddleston, M. J.; Verma, R.; Deshaies, R. J.; Carr, S. A. A multidimensional electrospray MS-based approach to phosphopeptide mapping. Anal. Chem. 2001, 73, 393-404. 